\title{
Transesophageal Echocardiography Probe Induced Recurrent VF In A Child with Severe RV Dysfunction. Report of a Rare Case
}

\author{
Vishnu Datt ${ }^{1}$, Sanjula Virmani ${ }^{2}$, Rachna Wadhwa ${ }^{1}$, Saket Agarwal ${ }^{1}$, Shardha Malik ${ }^{1}$, and \\ Varun Sharma ${ }^{1}$ \\ ${ }^{1}$ GB Pant Hospital \\ ${ }^{2} \mathrm{G}$ B Pant Hospital
}

May 14, 2021

\begin{abstract}
The safety of transesophageal echocardiography (TEE) probes has been documented in pediatric patients (neonates, infants, and small children even $<2.5 \mathrm{Kg}$ ). The overall safety profile of TEE probe is quite favourable with a reported incidence of complications is about $1-3 \%$. However, insertion of the TEE probe can induce vagal and sympathetic reflexes such as hypertension or hypotension, non-sustained ventricular and supraventricular tachyarrhythmias or bradyarrhythmias [3rd degree heart block], and even angina and myocardial infarction. We hereby document a repeated and fatal intraoperative VF precipitated by TEE probe in a 2-year-old, $10 \mathrm{~kg}$ paediatric patient diagnosed with ostium secundum atrial septal defect (OS-ASD), supravalvular pulmonary stenosis (PS) and severe right ventricular (RV) dysfunction.
\end{abstract}

\section{Introduction:}

Perioperative TEE is a critically important cardiovascular imaging modality. However, TEE in the pediatric patients became widespread after the introduction of miniaturized probes in the late 1980s.[1] It has a major role in hemodynamic management and detecting residual lesions during and/or after pediatric cardiac surgery. The overall safety profile of TEE probe is quite favourable with a reported incidence of complications is about 1-3\%.[2] Athough, the safety of TEE probes has been documented in pediatric patients with small weight, complications such as airway obstruction (1\%), right main stem advancement of the endotracheal tube $(0.2 \%)$, tracheal extubation (0.5\%), and vascular compression $(0.6 \%)$, esophageal perforation and bleeding have been reported. [3] Most of the problems seen in neonates and small infants are associated with respiratory compromise or vascular compression.[4] However, repeated ventricular fibrillation (VF) precipitated by TEE probe has not been described in the literature till date. We hereby report a recurrent and fatal intraoperative VF precipitated by TEE probe in a 2-year-old, $10 \mathrm{~kg}$ paediatric patient with OS-ASD, supravalvular PS and severe RV dysfunction.

Key words: Recurrent VF, TEE probe, RV dysfunction, Biphasic DC shock

\section{Case Report:}

A 2- years- old, $10 \mathrm{~kg}$, female child presented with bluish discolouration on crying since one and a half months of age. Transthoracic echocardiography (TTE) revealed a large OS-ASD with bidirectional shunt, supravalvular and infundibular PS with peak gradient of $120 \mathrm{mmHg}$, mild right RV dysfunction (TAPSE$13 \mathrm{~mm}$ ). The patient underwent a balloon dilatation for infundibular and supravalvular PS at the age of 5 months, which provided some relief from the symptoms. However, when she again started having bluish discolouration of lips and fingertips on crying, she was referred to CTVS unit for intra-cardiac repair. Following confirmation of first and fifth day negative RTPCR reports for Corona Sars Virus 19, and obtaining the 
Informed consent from the parents, she was taken up for intra-cardiac surgical repair under cardiopulmonary bypass (CPB).

In the OR, her base line $\mathrm{SpO} 2$ was $92 \%$ on air. The usual opioids-based anesthesia technique was used similar to any high-risk patients with complex congenital heart disease. In addition to standard ASA monitoring, TEE was also performed using paediatric probe (Size- 11x8mm, Model- GE VIVID S60 N), which confirmed TTE findings, and severe tricuspid regurgitation (TR) with jet reaching to left atrium (LA) through large ASD suggestive of extremely high right atrial (RA) pressures (CVP $=22 \mathrm{mmHg}$ ).(Fig. 1, Video 1) RV was severely dilated, almost akinetic, and interventricular septum was seen to be shifted towards the left ventricle (LV). In contrast, LV was D-shaped, exceedingly small and hyper-contractile (lateral wall was touching septum during systole) with LV ejection fraction of 80\%. (Video 1,2) (Fig.1,2). However, the patient developed VF before applying the chest incision while the TEE probe was advanced from oesophagus to transgastric position. It was successfully managed with intravenous lignocaine (15 mg), external monophasic DC shock (50 Joules), and followed by open cardiac massage and with internal biphasic DC shock (7 Joules) after rapid sternotomy. Concomitantly, it was decided to go on CPB and so, heparin (300 units $/ \mathrm{kg}$ ) was used as an anticoagulant and after confirming of an ACT value $>480$ seconds, standard CPB technique with moderate hypothermia (27.5 degrees C) and antegrade blood cardioplegic (Del Nido) myocardial protection was used for intra - cardiac repair. The cardiac repair involved as pericardial patch closure of OS -ASD, right ventricule outflow tract (RVOT) muscle bundle resection, non- transannular pericardial patch augmentation of RVOT and main pulmonary artery (MPA). In view of severe RV dysfunction, a PFO of $0.75 \mathrm{~cm}$ was also created.

After rewarming, the patient had a normal sinus rhythm (124 bpm) and was successfully weaned off CPB using infusion of Adrenaline (.05ug $/ \mathrm{kg} / \mathrm{min})$, Milrinone $(0.5 \mathrm{ug} / \mathrm{kg} / \mathrm{min})$, NTG $(1 \mathrm{mic} / \mathrm{kg} / \mathrm{min})$ and Noradrenaline $(.05 \mathrm{ug} / \mathrm{kg} / \mathrm{min})$. However, during post- operative TEE evaluation patient again developed two episodes of VF, which were reverted to normal sinus rhythm without any residual ischemic changes (Normal $\mathrm{ST}, \mathrm{T}$ waves) with the use of lignocaine and internal biphasic DC shock $(7 \mathrm{~J})$. In view of poor haemodynamic status [systolic blood pressure (SBP) - 68/27mmHg, CVP-14mmHg] and prolonged bypass time [CPB time $210 \mathrm{~min}$, Aortic cross clamp time 83 minutes], Modified ultrafiltration [MUF] was performed and total $800 \mathrm{cc}$ fluid was removed. However, once again she developed VF during MUF which was again managed successfully similar to the previous episodes. As the authors were not able to pin- point any other relevant cause for repeated VF [acidosis, electrolyte imbalance, ECG changes etc.], in the desperate scenario, the TEE probe was removed. Following this there occurred no repeated episode of VF. Following MUF there was a significant improvement in hemodynamics (BP -108/53mmHg, CVP-7mmHg, Hb- 16.6gm\%) which were maintained with moderate doses of inodilators. Patient was weaned off $\mathrm{CPB}$, heparin was neutralized with Protamine (1:1.3), and then the aorta was decannulated. Following adequate hemostasis, the chest was closed, and the patient was shifted to intensive care unit (ICU) for elective ventilation. During ICU stay, the patient required regular peritoneal dialysis for 3-4 days. Gradually, patient was weaned off inotropes and tracheal extubation was done on fifth post-operative day. Rest of the course was uneventful, and patient was shifted into the ward on $10^{\text {th }}$ post -operative day.

\section{Discussion:}

Perioperative TEE is a critically important cardiovascular imaging modality. The esophageal position of its transducer provides superior cardiac imaging and allows continuous intensive monitoring of the heart before, during, and after cardiac procedures. TEE performed immediately prior to the surgical intervention allows the operative team to review the preoperative diagnostic findings, identify any new findings, and assist in the assessment of hemodynamics and myocardial function prior to onset of the operative procedure. TEE may facilitate catheter placement, real-time monitoring of volume status and myocardial function to inform selection of anesthetic agents, inotropic support, and management decisions. [5,6,7] Overall use of the TEE in pediatric population is quite safe with a reported incidence of complications rate in cardiac surgical patients is approximately 1-3\%. [2,8] The Important complications are such as airway obstruction (1\%), right main stem advancement of the endotracheal tube $(0.2 \%)$, tracheal extubation $(0.5 \%)$, and vascular compression $(0.6 \%)$, 
esophageal perforation and even fatal gastrointestinal bleeding in 1.0\%. [3,9]. Most problems in neonates and small infants are associated with respiratory compromise or vascular compression.[4] TEE probe insertion can also induce cardiovascular complications through vagal and sympathetic reflexes like; hypertension or hypotension, tachyarrhythmias or bradycardia and even myocardial infarction. Often arrhythmias are manifested as non-sustained ventricular and supra ventricular tachy arrhythmias, atrial fibrillation, and 3rd degree heart block and can even induce angina and myocardial ischemia. [10,11] Pediatric patients may be more vulnerable to TEE, in that the esophageal probe can significantly compress vascular structures, such as a normally positioned or aberrant right subclavian artery, Pulmonary artery, the descending aorta,the innominate artery,and the pulmonary venous confluence in an infant with total anomalous pulmonary venous return. $[11,12]$

To the best of our knowledge the recurrent VF induced by TEE probe has not been described in the literature till date. The authors have documented the intraoperative recurrent VF precipitated by TEE probe in a pediatric patient with OS-ASD, sub valvular and supravalvular PS and severe RV dysfunction. Eventually the reasons that were not understood, the patient developed repeated VF with TEE probe movements. The TEE probe is much closer to the heart since the esophagus and heart are right next to each other. It has been hypothesized that the small sized oesophagus of child with TEE probe in situ became a rigid rod like structure, and as it lies just next to heart [RV, PA] that might have an exaggerated compressive effect on LV that was already compressed due to the distended, low compliant RV, and precipitated VF.[Fig 2] This mechanism has also been confirmed by easy reversibility with biphasic DC shock $[7 \mathrm{~J}]$ and excluding any residual ECG [ST/ T changes] and hemodynamics [systolic PB] effects prior or after development of VF, and reverting to the normal sinus rhythm following every episode of VF. Therefore, excluding the MI as a precipitating factor. It was also witnessed that mostly the VF episodes occurred during the forward insertion of the TEE probe from esophagus to trans-gastric or deep trans-gastric level and anteflexion. As the authors could not pin- point any other relevant cause for repeated VF, as a desperate attempt, the TEE probe was removed, following which the occurrence of repeated episodes of VF stopped immediately.

Conclusion : Paediatric TEE probe in young children may transform the oesophagus into a rigid rod like structure and may precipitate recurrent VF in paediatric patient with severe RV dysfunction causing leftward interventricular septal shifting and LV compression by further exaggerating the LV compressive effect.

\section{References:}

1. Weintraub R.Shiota T.Elkadi T.Golebiovski P.Zhang J.Rothman A.et al.Transesophageal echocardiography in infants and children with congenital heart disease.Circulation. 1992; 86: 711-722

2. Michael D. Puchalski, George K. Lui, Wanda C. Miller-Hance, Michael M. Luciana T. Young et al, Guidelines for Performing a Comprehensive Transesophageal Echocardiographic Examination in Children and All Patients with Congenital Heart Disease: Recommendations from the American Society of Echocardiography. Journal of the American Society of Echocardiography Volume 32 Number 2. Feb 2019;pp173-215

3. Abdulraouf M.Z.JijehaAhmad S.OmranabHani K.NajmabRiyadh M.Abu-Sulaiman. Role of intraoperative transesophageal echocardiography in pediatric cardiac surgery. J Saudi Heart Assoc2016;28:8994]

4. Andropoulos D, Ayres N, Stayer S, Bent S, Campos C, Fraser C. The effect of transesophageal echocardiography on ventilation in small infants undergoing cardiac surgery. Anesth Analg 2000;90:47-9.

5. Bai A, Steinberg M, Showler A, Burry L, Bhatia R, Tomlinson G, et al. Diagnostic accuracy of transthoracic echocardiography for infective endocarditis findings using transesophageal echocardiography as the reference standard: a meta-analysis. J Am Soc Echocardiogr 2017;30:639-46.e8.

6. Mart CR. Three-dimensional echocardiographic evaluation of the Fontan conduit for thrombus. Echocardiography 2012;29:363-8.

7. Saric M, Armour AC, Arnaout MS, Chaudhry FA, Grimm RA, Kronzon I, et al. Guidelines for the use of echocardiography in the evaluation of a cardiac source of embolism. J Am Soc Echocardiogr 2016;29:1-42. 
8. Razvan Purza, BSc, Subhamay Ghosh, MD, PhD, Chris Walker, MD, Brett Hiebert, MS, Lillian Koley et al.Transesophageal Echocardiography Complications in Adult Cardiac Surgery: A Retrospective Cohort Study Ann Thorac Surg 2017;103:795-803

9. Lennon MJ, Gibbs NM, Weightman WM, Leber J, Ee HC, Yusoff IF. Transesophageal echocardiography-related gastrointestinal complications in cardiac surgical patients.J Cardiothorac Vasc Anesth. 2005; 19:141-145.

10. Goland S, Shimoni S, Attali M, et al. Fatal ventricular arrhythmia as a complication of transesophageal echocardiography. Eur J Echocardiogr. 2005;6:151-3.

11. Geneviève Côté * André Denault. Transesophageal echocardiography-related complications. can j anesth 2008 ;55: (9) :622-647.

12. Preisman S,Yusim Y, Mishali D, Perel A. Compression of the pulmonary artery during transesophageal echocardiography in a pediatric cardiac patient. Anesth Analg. 2003; 96 (table of contents): 85-87
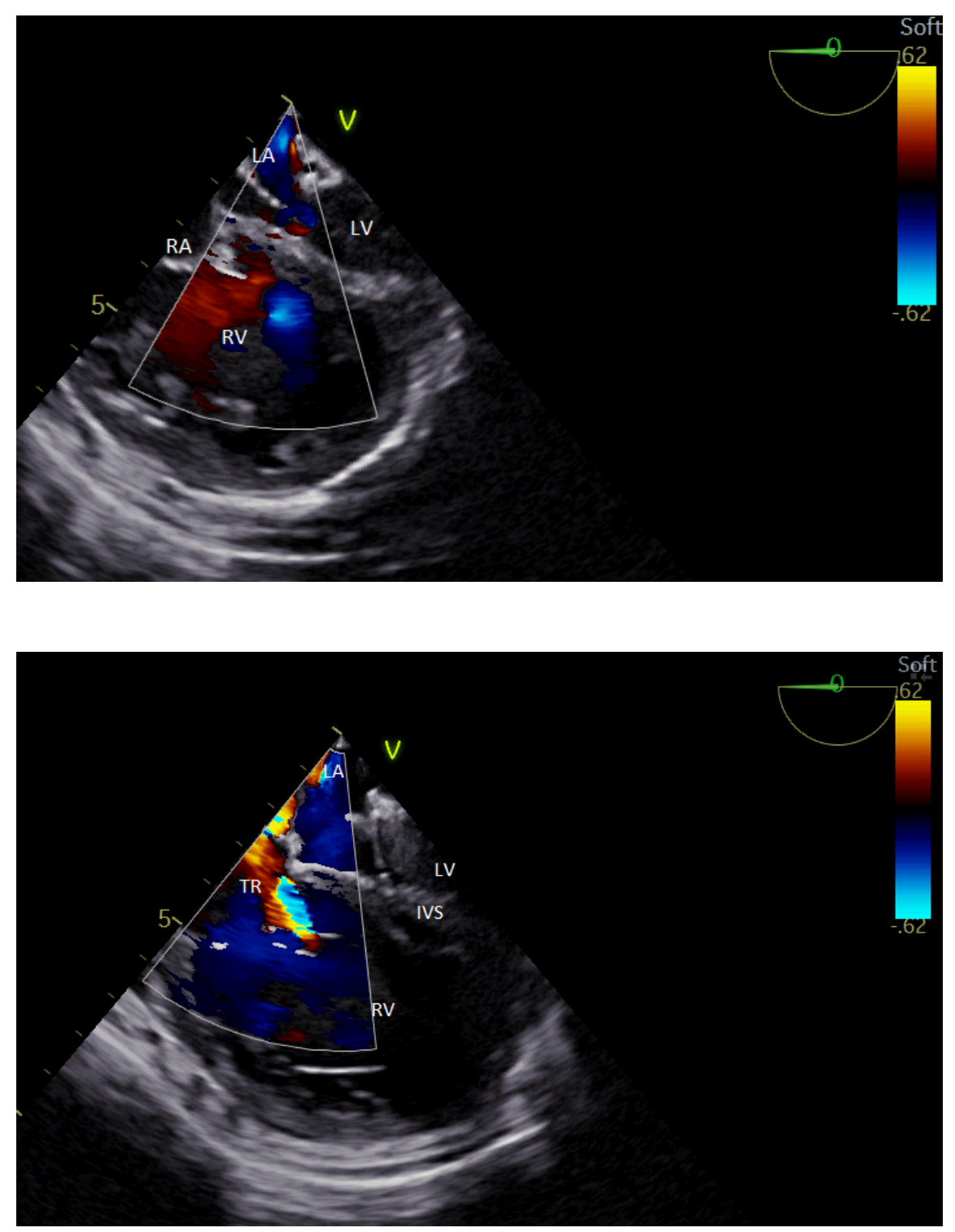

\section{Hosted file}


final_609ccf5e254507008c973f9a_831216.mp4 available at https://authorea.com/users/413861/ articles/522038-transesophageal-echocardiography-probe-induced-recurrent-vf-in-a-childwith-severe-rv-dysfunction-report-of-a-rare-case

\section{Hosted file}

final_609c181dcb1f300062032823_304929.mp4 available at https://authorea.com/users/413861/ articles/522038-transesophageal-echocardiography-probe-induced-recurrent-vf-in-a-childwith-severe-rv-dysfunction-report-of-a-rare-case 